\title{
The $\mathcal{S}$-cone and a primal-dual view on second-order representability
}

\author{
Helen Naumann ${ }^{1} \cdot$ Thorsten Theobald $^{1}$ \\ Received: 19 March 2020 / Accepted: 21 June 2020 / Published online: 8 July 2020 \\ (c) The Author(s) 2020
}

\begin{abstract}
The $\mathcal{S}$-cone provides a common framework for cones of polynomials or exponential sums which establish non-negativity upon the arithmetic-geometric inequality, in particular for sums of non-negative circuit polynomials (SONC) or sums of arithmeticgeometric exponentials (SAGE). In this paper, we study the $\mathcal{S}$-cone and its dual from the viewpoint of second-order representability. Extending results of Averkov and of Wang and Magron on the primal SONC cone, we provide explicit generalized secondorder descriptions for rational $\mathcal{S}$-cones and their duals.
\end{abstract}

Keywords Positive polynomials - Sums of non-negative circuit polynomials · Arithmetic-geometric exponentials $\cdot$ Dual cone $\cdot \mathcal{S}$-cone $\cdot$ Second-order cone

Mathematics Subject Classification $14 \mathrm{P} 10 \cdot 52 \mathrm{~A} 20 \cdot 90 \mathrm{C} 23$

\section{Introduction}

The question to characterize and to decide whether a polynomial or an exponential sum is non-negative occurs in many branches of mathematics and application areas. In the development of real algebraic geometry, the connection between the cone of non-negative polynomials and the cone of sums of squares of polynomials plays a prominent role (see, for example, Bochnak et al. 1998; Marshall 2008; Prestel and Delzell 2001). If a polynomial can be written as a sum of squares of polynomials, this provides a certificate for the non-negativity of the polynomial. Since the beginning of the current millennium, non-negativity certificates of polynomials have also seen much interest from the computational point of view and have strongly advanced the

$\triangle$ Thorsten Theobald

theobald@math.uni-frankfurt.de

Helen Naumann

naumann@math.uni-frankfurt.de

1 FB 12-Institut für Mathematik, Goethe-Universität, Postfach 11 19 32, 60054 Frankfurt am Main, Germany 
rich connections between real and convex algebraic geometry as well as polynomial optimization (see, for example, Lasserre 2010; Laurent 2009).

Within the research activities on non-negativity certificates in the last years, the cones of sums of arithmetic-geometric exponentials (SAGE, introduced by Chandrasekaran and Shah 2016) and sums of non-negative circuit polynomials (SONC, introduced by Iliman and de Wolff 2016) have received a lot of attention (see, e.g., Averkov 2019; Dressler et al. 2018a; Forsgård and de Wolff 2019; Murray et al. 2018, 2019; Wang 2018). These cones build upon earlier work of Reznick (1989). They provide non-negativity certificates based on the arithmetic-geometric inequality and are particularly useful in the context of sparse polynomials.

In Katthän et al. (2019), the authors of the current paper and Katthän have introduced a common generalization, called the $\mathcal{S}$-cone, which facilitates to study the SAGE cone and the SONC cone within a uniform generalized setting. Formally, for two finite disjoint sets $\emptyset \neq \mathcal{A} \subseteq \mathbb{R}^{n}, \mathcal{B} \subseteq \mathbb{N}^{n} \backslash(2 \mathbb{N})^{n}$, let $\mathbb{R}[\mathcal{A}, \mathcal{B}]$ denote the space of all functions $f: \mathbb{R}^{n} \rightarrow \mathbb{R} \cup\{\infty\}$ of the form

$$
f(\mathbf{x})=\sum_{\alpha \in \mathcal{A}} c_{\alpha}|\mathbf{x}|^{\alpha}+\sum_{\beta \in \mathcal{B}} c_{\beta} \mathbf{x}^{\beta} \in \mathbb{R}[\mathcal{A}, \mathcal{B}]
$$

with real coefficients $c_{\alpha}, \alpha \in \mathcal{A} \cup \mathcal{B}$. Our precondition $\mathcal{A} \cap \mathcal{B}=\emptyset$ is a slight restriction to the setup in Katthän et al. (2019), in order to enable a little more convenient notation.

One motivation for the class of functions (1.1) is that it allows to capture nonnegativity of polynomials on $\mathbb{R}^{n}$ and non-negativity of polynomials on the non-negative orthant $\mathbb{R}_{+}^{n}$ within a uniform setting. Moreover, global non-negativity of the summand $\sum_{\alpha \in \mathcal{A}} c_{\alpha}|\mathbf{x}|^{\alpha}$ is equivalent to global non-negativity of the exponential sum $\mathbf{y} \mapsto$ $\sum_{\alpha \in \mathcal{A}} c_{\alpha} \exp \left(\alpha^{T} \mathbf{y}\right)$

Definition 1.1 A function $f$ of the form (1.1) is called an even AG function if for at most one $\alpha \in \mathcal{A}, c_{\alpha}$ is negative and for all $\beta \in \mathcal{B}, c_{\beta}$ is zero; and it is called an odd $A G$ function if for all $\alpha \in \mathcal{A}, c_{\alpha}$ is non-negative and for at most one $\beta \in \mathcal{B}, c_{\beta}$ is nonzero.

$f$ is called an $A G$ function (arithmetic-geometric mean function), if $f$ is an even AG function or an odd AG function.

Definition 1.2 Let $\emptyset \neq \mathcal{A} \subseteq \mathbb{R}^{n}, \mathcal{B} \subseteq \mathbb{N}^{n} \backslash(2 \mathbb{N})^{n}$ be finite disjoint sets. The $\mathcal{S}$-cone $C_{\mathcal{S}}(\mathcal{A}, \mathcal{B})$ is defined as

$$
C_{\mathcal{S}}(\mathcal{A}, \mathcal{B}):=\text { cone }\{f \in \mathbb{R}[\mathcal{A}, \mathcal{B}]: f \text { is a non-negative } \mathrm{AG} \text { function }\}
$$

where cone denotes the conic (or positive) hull. $C_{\mathcal{S}}(\mathcal{A}, \mathcal{B})$ is called rational if $\mathcal{A} \subseteq \mathbb{Q}^{n}$.

The SAGE and SONC cones arise as special cases of this cone, see Sect. 2.

Both from the geometric and from the optimization point of view, it is of prominent interest to understand how the different classes of cones relate to each other and whether techniques for different cones can be fruitfully combined. Karaca et al. (2017) have studied non-negativity certificates based on a combination of the SAGE cone with the cone of sums of squares. Concerning relations between the various cones, Averkov has shown that the SONC cone can be represented as a projection of a spectrahedron 
(Averkov 2019). In fact, his proof applies the techniques from Ben-Tal and Nemirovski (2001), which reveals that the SONC cone is even second-order representable. Wang and Magron gave an alternative proof based on binomial squares and $\mathcal{A}$-mediated sets (Wang and Magron 2019).

Here, we take the general view of the $\mathcal{S}$-cone as well as a primal-dual viewpoint. Generalizing the results of Averkov and of Wang and Magron, we show that rational $\mathcal{S}$-cones and their duals are second-order representable and provide explicit and direct descriptions. Our proof combines the techniques for the second-order cones techniques from Ben-Tal and Nemirovski (2001) with the concepts and the duality theory of the $\mathcal{S}$ cone from Katthän et al. (2019). Our derivation is different from the approach of Wang and Magron, and it does not need binomial squares or $\mathcal{A}$-mediated sets. Moreover, our second-order representation prevents the consideration of redundant circuits by using a characterization of the extreme rays of the $\mathcal{S}$-cone from Katthän et al. (2019).

Beyond the specific representability result, the goal of the paper is to offer further insights into the use of the framework of the $\mathcal{S}$-cone as a generalization of SONC and SAGE.

\section{Preliminaries}

Throughout the text, we use the notations $\mathbb{N}=\{0,1,2,3, \ldots\}$ and $\mathbb{R}_{+}=\{x \in \mathbb{R}$ : $x \geq 0\}$. For a finite subset $\mathcal{A} \subseteq \mathbb{R}^{n}$, denote by $\mathbb{R}^{\mathcal{A}}$ the set of $|\mathcal{A}|$-dimensional vectors whose components are indexed by the set $\mathcal{A}$. Moreover, we write

$$
|\mathbf{x}|^{\alpha}=\prod_{j=1}^{n}\left|x_{j}\right|^{\alpha_{j}} \quad \text { and } \quad \mathbf{x}^{\beta}=\prod_{j=1}^{n} x_{j}^{\beta_{j}},
$$

and if one component of $\mathbf{x}$ is zero and the corresponding exponent is negative, then we set $|\mathbf{x}|^{\alpha}=\infty$.

\subsection{The $\mathcal{S}$-cone, SAGE and SONC}

We explain that the $\mathcal{S}$-cone generalizes the SAGE cone and the SONC cone and collect some basic properties of the three cones.

The SAGE cone Let $\mathcal{A}$ be a non-empty, finite set. An exponential sum supported on $\mathcal{A}$ is a function of the form

$$
\mathbf{y} \mapsto \sum_{\alpha \in \mathcal{A}} c_{\alpha} \exp \left(\alpha^{T} \mathbf{y}\right)
$$

with real coefficients $c_{\alpha}$. If $\mathcal{B}=\emptyset$, then $\mathbb{R}[\mathcal{A}, \mathcal{B}]$ can be identified with the space of exponential sums supported on $\mathcal{A}$ by means of the substitution $\left|x_{i}\right|=\exp \left(y_{i}\right)$.

For finite $\mathcal{A} \subseteq \mathbb{R}^{n}, \mathcal{A}^{\prime} \subsetneq \mathcal{A}$ and $\beta \in \mathcal{A} \backslash \mathcal{A}^{\prime}$, the $\operatorname{SAGE}$ cone $C_{\mathrm{SAGE}}(\mathcal{A})$ is defined as

$$
C_{\mathrm{SAGE}}(\mathcal{A})=\sum_{\beta \in \mathcal{A}} C_{\mathrm{AGE}}(\mathcal{A} \backslash\{\beta\}, \beta),
$$


where for $\mathcal{A}^{\prime}:=\mathcal{A} \backslash\{\beta\}$

$$
\begin{aligned}
& C_{\mathrm{AGE}}\left(\mathcal{A}^{\prime}, \beta\right)=\left\{c \in \mathbb{R}^{\mathcal{A}}: c_{\alpha} \geq 0 \text { for } \alpha \in \mathcal{A}^{\prime},\right. \\
& \left.\sum_{\alpha \in \mathcal{A}^{\prime}} c_{\alpha} \exp \left(\alpha^{T} x\right)+c_{\beta} \exp \left(\beta^{T} x\right) \geq 0 \text { on } \mathbb{R}^{n}\right\}
\end{aligned}
$$

(see Chandrasekaran and Shah 2016). We observe that the $\mathcal{S}$-cone $C_{\mathcal{S}}(\mathcal{A}, \varnothing)$ can be identified with $C_{\mathrm{SAGE}}(\mathcal{A})$ using the substitution $(2.1) . C_{\mathrm{SAGE}}(\mathcal{A})$ is a closed convex cone in $\mathbb{R}^{\mathcal{A}}$. The membership problem for this convex cone can be formulated as a relative entropy program (Murray et al. 2018, see also Proposition 2.2 below).

The SONC cone Here, let the non-empty finite set $\mathcal{A}$ be contained in $\mathbb{N}^{n}$. Let

$$
I(\mathcal{A})=\left\{(A, \beta): A \subseteq(2 \mathbb{N})^{n} \cap \mathcal{A} \text { affinely independent, } \beta \in \operatorname{relint}(\operatorname{conv} A) \cap \mathcal{A}\right\},
$$

where relint denotes the relative interior of a set. For singleton sets $A=\{\alpha\}$, the sets $(A, \beta)$ are formally of the form $(\{\alpha\}, \alpha)$. By convention, we write these circuits simply as $(\alpha)$, and with this convention, the set $\left\{(\alpha): \alpha \in(2 \mathbb{N})^{n}\right\} \cap \mathcal{A}$ is contained in $I(\mathcal{A})$.

For $(A, \beta) \in I(\mathcal{A})$, let $P_{A, \beta}$ denote the set of polynomials in $\mathbb{R}\left[x_{1}, \ldots, x_{n}\right]$ whose supports are contained in $A \cup\{\beta\}$ and which are non-negative on $\mathbb{R}^{n}$. The Minkowski sum

$$
C_{\mathrm{SONC}}(\mathcal{A})=\sum_{(A, \beta) \in I(\mathcal{A})} P_{A, \beta}
$$

defines the cone of SONC polynomials with support $\mathcal{A}$ (see Averkov 2019; Iliman and de Wolff 2016).

The cone $C_{\mathrm{SONC}}(\mathcal{A})$ is a closed convex cone, and it can be recognized as a special case of a rational $\mathcal{S}$-cone by observing

$$
C_{\mathrm{SONC}}(\mathcal{A})=C_{\mathcal{S}}\left(\mathcal{A} \cap(2 \mathbb{N})^{n}, \mathcal{A} \cap\left(\mathbb{N}^{n} \backslash(2 \mathbb{N})^{n}\right)\right)
$$

(see Katthän et al. 2019). Using the results from Murray et al. (2018), membership in the SONC cone can also be formulated in terms of a relative entropy program.

The $\mathcal{S}$-cone The $\mathcal{S}$-cone from Definition 1.1 offers a uniform setting for the SAGE and the SONC cones. We collect some further properties of the $\mathcal{S}$-cone. For a nonempty finite set $\mathcal{A} \subseteq \mathbb{R}^{n}$ and $\beta \in \mathbb{N}^{n} \backslash\left((2 \mathbb{N})^{n} \cup \mathcal{A}\right)$ let

$$
P_{\mathcal{A}, \beta}^{\text {odd }}:=\left\{f: f=\sum_{\alpha \in \mathcal{A}} c_{\alpha}|\mathbf{x}|^{\alpha}+c_{\beta} \mathbf{x}^{\beta}, f(\mathbf{x}) \geq 0 \forall \mathbf{x} \in \mathbb{R}^{n}, c_{\mid \mathcal{A}} \in \mathbb{R}_{+}^{\mathcal{A}}, c_{\beta} \in \mathbb{R}\right\}
$$


be the cone of non-negative odd AG functions supported on $(\mathcal{A}, \beta)$, and similarly for $\beta \in \mathbb{R}^{n} \backslash \mathcal{A}$ let

$$
P_{\mathcal{A}, \beta}^{\text {even }}:=\left\{f: f=\sum_{\alpha \in \mathcal{A}} c_{\alpha}|\mathbf{x}|^{\alpha}+c_{\beta}|\mathbf{x}|^{\beta}, f(\mathbf{x}) \geq 0 \forall \mathbf{x} \in \mathbb{R}^{n}, c_{\mid \mathcal{A}} \in \mathbb{R}_{+}^{\mathcal{A}}, c_{\beta} \in \mathbb{R}\right\}
$$

be the cone of non-negative even AG functions supported on $(\mathcal{A}, \beta)$. By definition,

$$
C_{\mathcal{S}}(\mathcal{A}, \mathcal{B})=\sum_{\alpha \in \mathcal{A}} P_{\mathcal{A} \backslash\{\alpha\}, \alpha}^{\text {even }}+\sum_{\beta \in \mathcal{B}} P_{\mathcal{A}, \beta}^{\text {odd }}
$$

Note that non-negative even AG functions correspond exactly to the AGE functions (arithmetic-geometric exponentials) in Chandrasekaran and Shah (2016) and Murray et al. (2018).

The following alternative representation allows to express the $\mathcal{S}$-cone in terms of the SAGE cone. Here, $|d|$ denotes the absolute value of the vector $d \in \mathbb{R}^{\mathcal{B}}$, taken component-wise.

Proposition 2.1 (Katthän et al. 2019) Let $\emptyset \neq \mathcal{A} \subseteq \mathbb{R}^{n}, \mathcal{B} \subseteq \mathbb{N}^{n} \backslash(2 \mathbb{N})^{n}$ be finite and disjoint. Then,

$$
\begin{aligned}
C_{\mathcal{S}}(\mathcal{A}, \mathcal{B}) & =\left\{\sum_{\alpha \in \mathcal{A}} c_{\alpha}|x|^{\alpha}+\sum_{\beta \in \mathcal{B}} d_{\beta} x^{\beta}:(c,-|d|) \in C_{\mathrm{SAGE}}(\mathcal{A} \cup \mathcal{B})\right\} \\
& =\left\{\sum_{\alpha \in \mathcal{A}} c_{\alpha}|x|^{\alpha}+\sum_{\beta \in \mathcal{B}} d_{\beta} x^{\beta}: \exists t \in \mathbb{R}^{\mathcal{B}}(c, t) \in C_{\mathrm{SAGE}}(\mathcal{A} \cup \mathcal{B}), t \leq-|d|\right\} .
\end{aligned}
$$

For a finite set $\emptyset \neq \mathcal{A} \subseteq \mathbb{R}^{n}$, we use the notion

$$
C_{\mathcal{S}}(\mathcal{A}):=C_{\mathcal{S}}(\mathcal{A}, \emptyset)=C_{\mathrm{SAGE}}(\mathcal{A})
$$

and immediately observe $C_{\mathcal{S}}(\mathcal{A})=\sum_{\alpha \in \mathcal{A}} P_{\mathcal{A} \backslash\{\alpha\}, \alpha}^{\text {even }}$. Hence, for our purpose it suffices to study the cone $P_{\mathcal{A}, \beta}^{\text {even }}$ of even AG functions and use the results of this cone for the odd case in Sect. 4.

Using the relative entropy function and the circuit number, the cones $P_{\mathcal{A}, \beta}^{\text {even }}$ and $P_{\mathcal{A}, \beta}^{\text {odd }}$ can be characterized in terms of convex optimization problems. For a finite set $\emptyset \neq \mathcal{A} \subseteq \mathbb{R}^{n}$, denote by $D: \mathbb{R}_{>0}^{\mathcal{A}} \times \mathbb{R}_{>0}^{\mathcal{A}} \rightarrow \mathbb{R}$,

$$
D(v, \gamma)=\sum_{\alpha \in \mathcal{A}} v_{\alpha} \ln \left(\frac{v_{\alpha}}{\gamma_{\alpha}}\right)
$$

the relative entropy function. $D$ can also be extended to $\mathbb{R}_{+}^{\mathcal{A}} \times \mathbb{R}_{+}^{\mathcal{A}} \rightarrow \mathbb{R} \cup\{\infty\}$ via the conventions $0 \cdot \ln \frac{0}{y}=0$ for $y \geq 0$ and $y \cdot \ln \frac{y}{0}=\infty$ for $y>0$. Non-negativity of an 
(even or odd) AG function $f$ with coefficients $c_{\alpha}$ and $c_{\beta}$ can be characterized through the product $\prod_{\alpha \in \mathcal{A}}\left(c_{\alpha} / \lambda_{\alpha}\right)^{\lambda_{\alpha}}$ and $c_{\beta}$ (see Katthän et al. 2019, Theorem 2.7). For an affinely independent ground set $\mathcal{A}$, this product is called the circuit number of $f$ (see Iliman and de Wolff 2016). In particular, for an even AG function, this non-negativity characterization in terms of the circuit number is given by

$$
\prod_{\alpha \in \mathcal{A}}\left(\frac{c_{\alpha}}{\lambda_{\alpha}}\right)^{\lambda_{\alpha}} \geq-c_{\beta} .
$$

The following characterization of $P_{\mathcal{A}, \beta}^{\text {even }}$ and $P_{\mathcal{A}, \beta}^{\text {odd }}$ in terms of the relative entropy function and in terms of the circuit number is a direct consequence of Theorem 2.7 of Katthän et al. (2019).

Proposition 2.2 Let $\mathcal{A} \subseteq \mathbb{R}^{n}$ be a non-empty finite set, $\beta \in \mathbb{R}^{n} \backslash \mathcal{A}$ and an AG function $f$ with coefficient vector c supported on $\mathcal{A} \cup\{\beta\}$.

1. If $f$ is an even $A G$ function, then

$$
\begin{aligned}
f \in P_{\mathcal{A}, \beta}^{\text {even }} & \Longleftrightarrow \exists v \in \mathbb{R}_{+}^{\mathcal{A}} \sum_{\alpha \in \mathcal{A}} v_{\alpha} \alpha=\left(\sum_{\alpha \in \mathcal{A}} v_{\alpha}\right) \beta, D(v, e \cdot c) \leq c_{\beta} \\
& \Longleftrightarrow \exists \lambda \in \mathbb{R}_{+}^{\mathcal{A}} \sum_{\alpha \in \mathcal{A}} \lambda_{\alpha} \alpha=\beta, \sum_{\alpha \in \mathcal{A}} \lambda_{\alpha}=1, \prod_{\alpha \in \mathcal{A}}\left(\frac{c_{\alpha}}{\lambda_{\alpha}}\right)^{\lambda_{\alpha}} \geq-c_{\beta} .
\end{aligned}
$$

2. If $f$ is an odd AG function, then

$$
\begin{aligned}
f \in P_{\mathcal{A}, \beta}^{\text {odd }} & \Longleftrightarrow \exists v \in \mathbb{R}_{+}^{\mathcal{A}} \sum_{\alpha \in \mathcal{A}} v_{\alpha} \alpha=\left(\sum_{\alpha \in \mathcal{A}} v_{\alpha}\right) \beta, D(v, e \cdot c) \leq-\left|c_{\beta}\right| \\
& \Longleftrightarrow \exists \lambda \in \mathbb{R}_{+}^{\mathcal{A}} \sum_{\alpha \in \mathcal{A}} \lambda_{\alpha} \alpha=\beta, \sum_{\alpha \in \mathcal{A}} \lambda_{\alpha}=1, \prod_{\alpha \in \mathcal{A}}\left(\frac{c_{\alpha}}{\lambda_{\alpha}}\right)^{\lambda_{\alpha}} \geq\left|c_{\beta}\right| .
\end{aligned}
$$

If $\mathcal{A}$ is a set of affinely independent vectors and $\beta \in \operatorname{relint} \mathcal{A}$, then $\lambda$ is unique. We call the corresponding AG function a circuit function, the tuple $(\mathcal{A}, \beta)$ the circuit and identify the unique $\lambda$ with the above declared characteristics $\lambda=\lambda(\mathcal{A}, \beta)$.

\subsection{Duality theory}

Studying the duality theory has been initiated in Chandrasekaran and Shah (2016) (for SAGE), Dressler et al. (2018b) (for SONC) and Katthän et al. (2019) (for the $\mathcal{S}$ cone). See also the recent work of Papp (2019), who developed an alternative approach for deriving the dual cones, by expressing the non-negativity of circuit polynomials in terms of a power cone. We can identify the dual space of $\mathbb{R}[\mathcal{A}]$ with $\mathbb{R}^{\mathcal{A}}$. For $f \in \mathbb{R}[\mathcal{A}]$ with coefficients $\mathbf{c} \in \mathbb{R}^{\mathcal{A}}$ and an element $\mathbf{v} \in \mathbb{R}^{\mathcal{A}}$, we consider the natural duality pairing 


$$
\mathbf{v}(f)=\sum_{\alpha \in \mathcal{A}} v_{\alpha} c_{\alpha}
$$

Using this notation, the dual cone $\left(C_{\mathcal{S}}(\mathcal{A})\right)^{*}$ is defined as

$$
\left(C_{\mathcal{S}}(\mathcal{A})\right)^{*}=\left\{\mathbf{v} \in \mathbb{R}^{\mathcal{A}}: \mathbf{v}(f) \geq 0 \text { for all } f \in C_{\mathcal{S}}(\mathcal{A})\right\} .
$$

The following statement expresses the dual $\mathcal{S}$-cone in terms of the dual SAGE cone.

Proposition 2.3 Let $\emptyset \neq \mathcal{A} \subseteq \mathbb{R}^{n}$ and $\mathcal{B} \subseteq \mathbb{N}^{n} \backslash(2 \mathbb{N})^{n}$ disjoint and finite. The dual cone of the $\mathcal{S}$-cone $C_{\mathcal{S}}(\mathcal{A}, \mathcal{B})$ is

$$
\begin{aligned}
C_{\mathcal{S}}(\mathcal{A}, \mathcal{B})^{*} & =\left\{(\mathbf{v}, \mathbf{w}) \in \mathbb{R}^{\mathcal{A}} \times \mathbb{R}^{\mathcal{B}}:(\mathbf{v},|\mathbf{w}|) \in C_{S A G E}(\mathcal{A} \cup \mathcal{B})^{*}\right\} \\
& =\left\{(\mathbf{v}, \mathbf{w}) \in \mathbb{R}^{\mathcal{A}} \times \mathbb{R}^{\mathcal{B}}: \exists \mathbf{u} \in \mathbb{R}^{\mathcal{B}}(\mathbf{v}, \mathbf{u}) \in C_{S A G E}(\mathcal{A} \cup \mathcal{B})^{*}, \mathbf{u} \geq|\mathbf{w}|\right\} .
\end{aligned}
$$

Proof We use (2.5), which provides a characterization for the primal cone $C_{\mathcal{S}}(\mathcal{A}, \mathcal{B})$ in terms of an existential quantification. Consider its lifted cone

$$
\begin{aligned}
\widehat{C_{\mathcal{S}}}(\mathcal{A}, \mathcal{B}) & :=C_{\mathrm{SAGE}}(\mathcal{A} \cup \mathcal{B}) \times \mathbb{R}^{\mathcal{B}} \cap\left\{(\mathbf{c}, \mathbf{t}, \mathbf{d}): t_{\beta} \leq-\left|d_{\beta}\right| \text { for all } \beta \in \mathcal{B}\right\} \\
& =C_{\mathrm{SAGE}}(\mathcal{A} \cup \mathcal{B}) \times \mathbb{R}^{\mathcal{B}} \cap\left\{(\mathbf{c}, \mathbf{t}, \mathbf{d}): t_{\beta} \leq d_{\beta}, t_{\beta} \leq-d_{\beta} \text { for all } \beta \in \mathcal{B}\right\}
\end{aligned}
$$

in the space $\mathbb{R}^{\mathcal{A}} \times \mathbb{R}^{\mathcal{B}} \times \mathbb{R}^{\mathcal{B}}$. The dual cone of the right-hand cone in (2.10) is the set

$$
\text { cone }\left\{\left(0, \ldots, 0,-e^{(\beta)}, \pm e^{(\beta)}\right): \beta \in \mathcal{B}\right\}
$$

where $e^{(\beta)}$ denotes the unit vector with respect to $\beta \in \mathcal{B}$. As intersection and Minkowski sum are dual operations, we obtain

$$
\widehat{C_{\mathcal{S}}}(\mathcal{A}, \mathcal{B})^{*}=C_{\mathrm{SAGE}}(\mathcal{A} \cup \mathcal{B})^{*} \times\{0\}+\operatorname{cone}\left\{\left(0, \ldots, 0,-e^{(\beta)}, \pm e^{(\beta)}\right): \beta \in \mathcal{B}\right\}
$$

Identifying the $\mathcal{S}$-cone with its coefficients, we can express $C_{\mathcal{S}}(\mathcal{A}, \mathcal{B})^{*}$ in terms of the lifted cone $\widehat{C_{\mathcal{S}}}(\mathcal{A}, \mathcal{B})$ by

$$
C_{\mathcal{S}}(\mathcal{A}, \mathcal{B})^{*}=\widehat{C_{\mathcal{S}}}(\mathcal{A}, \mathcal{B}) \cap\left\{(\mathbf{v}, \mathbf{s}, \mathbf{w}) \in \mathbb{R}^{\mathcal{A}} \times \mathbb{R}^{\mathcal{B}} \times \mathbb{R}^{\mathcal{B}}: \mathbf{s}=0\right\}
$$

Thus, $(\mathbf{v}, \mathbf{w}) \in C_{\mathcal{S}}(\mathcal{A}, \mathcal{B})$ whenever $(\mathbf{v},|\mathbf{w}|) \in C_{\mathrm{SAGE}}(\mathcal{A} \cup \mathcal{B})^{*}$. Convexity then implies the second characterization (2.9).

Hence, as in the primal case, it suffices to study even AG functions in the dual situation. We will make use of a representation of the dual of the $\mathcal{S}$-cone from Katthän et al. (2019). For this, observe that similar to the SONC case in (2.2), one can also 
Fig. 1 Circuit $(A, \beta)$

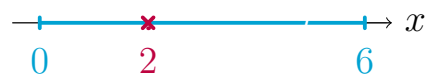

Fig. 2 Circuit $\left(A^{\prime}, \beta^{\prime}\right)$

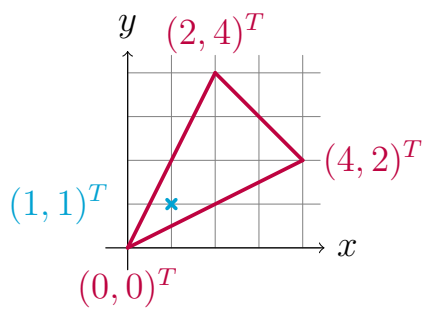

consider circuits in the case of the SAGE cone. In slight variation of (2.2), for a finite set $\emptyset \neq \mathcal{A} \subseteq \mathbb{R}^{n}$, the set of circuits supported on $\mathcal{A}$ is the set

$$
I(\mathcal{A})=\{(A, \beta): A \subseteq \mathcal{A} \text { affinely independent, } \beta \in \operatorname{relint}(\operatorname{conv} A) \cap(\mathcal{A} \backslash A)\} .
$$

Two examples of circuits are the pairs $(A, \beta)$ with $A=\{0,6\}$ and $\beta=\{2\}$ (see Fig. 1) and $\left(A^{\prime}, \beta^{\prime}\right)$ with $A^{\prime}=\left\{(0,0)^{T},(4,2)^{T},(2,4)^{T}\right\}$ and $\beta^{\prime}=(1,1)^{T}$ (see Fig. 2).

Thereby, the dual $\mathcal{S}$-cone $C_{\mathcal{S}}(\mathcal{A})$ can be represented as follows.

Proposition 2.4 (Katthän et al. 2019, Theorem 3.5) Let $\emptyset \neq \mathcal{A} \subseteq \mathbb{R}^{n}$ be finite. Then a point $\mathbf{v} \in \mathbb{R}^{\mathcal{A}}$ is contained in $C_{\mathcal{S}}(\mathcal{A})^{*}$ if and only if $\mathbf{v} \geq 0$ and

$$
\ln \left(v_{\beta}\right) \leq \sum_{\alpha \in A} \lambda_{\alpha} \ln \left(v_{\alpha}\right) \text { for every circuit }(A, \beta) \text { in } I(\mathcal{A}) \text { and } \lambda=\lambda(A, \beta)
$$

\subsection{Second-order formulations}

Let $[m]$ abbreviate the set $\{1, \ldots, m\}$ and denote by $\|\cdot\|$ the Euclidean norm. A second-order cone program (SOCP) is an optimization problem of the form

$$
\min \left\{\mathbf{c}^{T} \mathbf{x}:\left\|A_{i} \mathbf{x}+\mathbf{b}_{i}\right\|_{2} \leq \mathbf{c}_{i}^{T} \mathbf{b}+\mathbf{d}_{i} \text { for all } i \in[m]\right\}
$$

with real symmetric matrices $A_{i}$, vectors $\mathbf{b}_{i}, \mathbf{c}_{i}, \mathbf{d}_{i}$ and a vector $\mathbf{c}$. A subset of $\mathbb{R}^{n}$ is called second-order representable if it can be represented as a projection of the feasible set of a second-order program.

For a symmetric $2 \times 2$-matrix, positive semidefiniteness can be formulated as a second-order condition. 
Lemma 2.5 (See, e.g., Nesterov and Nemirovski 1994, §6.4.3.8, Wang and Magron 2019, Lemma 4.3) A symmetric $2 \times 2$ matrix $A=\left(\begin{array}{ll}a & b \\ b & c\end{array}\right)$ is positive semidefinite if and only if the second-order condition

$$
\left\|\left(\begin{array}{c}
2 b \\
a-c
\end{array}\right)\right\|_{2} \leq a+c
$$

is satisfied.

Let $S_{+}^{n}$ be the subset of symmetric $n \times n$-matrices which are positive semidefinite. By Averkov (2019), there exists some $m \in \mathbb{N}$ so that the cone of SONC polynomials $C_{\text {SONC }}(\mathcal{A})$ supported on $\mathcal{A}$ can be written as the projection of the spectrahedron $\left(S_{+}^{2}\right)^{m} \cap H$ for some affine space $H$.

\section{A second-order representation for the cone of non-negative AG functions and its dual}

In order to provide a second-order representation for the $\mathcal{S}$-cone and its dual, the main task is to capture the cone of non-negative AG functions and its dual. For a comprehensive collection of techniques for handling second-order cones, we refer to Ben-Tal and Nemirovski (2001).

Throughout the section, let $(A, \beta)$ be a fixed circuit and rational barycentric coordinates $\lambda \in \mathbb{R}_{+}^{A}$, which represent $\beta$ as a convex combination of $A$. That is, $\beta=\sum_{\alpha \in \mathcal{A}} \lambda_{\alpha} \alpha$ and $\sum_{\alpha \in \mathcal{A}} \lambda_{\alpha}=1$. Let $p \in \mathbb{N}$ denote the smallest common denominator of the fractions $\lambda_{\alpha}$ for $\alpha \in A$, i.e., $\lambda_{\alpha}=\frac{p_{\alpha}}{p}$ with $p_{\alpha} \in \mathbb{N}$ for all $\alpha \in A$ and $p$ is minimal.

With the given circuit $(A, \beta) \in I(\mathcal{A})$, we associate a set of dual circuit variables

$$
\left(y_{k, i}\right)_{k, i}
$$

where $k \in\left[\left\lceil\log _{2}(p)\right\rceil-1\right]$ and $i \in\left[2^{\left\lceil\log _{2}(p)\right\rceil-k}\right]$. The collection of these $\sum_{k=1}^{\left\lceil\log _{2}(p)\right\rceil-1} 2^{\left\lceil\log _{2}(p)\right\rceil-k}=2^{\left\lceil\log _{2}(p)\right\rceil}-2$ variables is denoted as $\mathbf{y}^{A, \beta}$ or shortly as $\mathbf{y}$. Further, denote the restriction of a vector $\mathbf{v} \in \mathbb{R}^{\mathcal{A}}$ to the components of $A \subseteq \mathcal{A}$ by $\mathbf{v}_{\mid A}$.

Definition 3.1 A dual circuit matrix $C_{A, \beta}^{*}\left(\mathbf{v}_{\mid A}, v_{\beta}, \mathbf{y}\right)$ is a block diagonal matrix consisting of the blocks

$$
\begin{aligned}
& \left(\begin{array}{cc}
y_{k-1,2 i-1} & y_{k, i} \\
y_{k, i} & y_{k-1,2 i}
\end{array}\right) \text { for } k \in\left\{2, \ldots,\left\lceil\log _{2}(p)\right\rceil-1\right\} \text { and } i \in\left[2^{\left\lceil\log _{2}(p)\right\rceil-k}\right], \\
& \left(\begin{array}{cc}
y_{\left\lceil\log _{2}(p)\right\rceil-1,1} & v_{\beta} \\
v_{\beta} & y_{\left\lceil\log _{2}(p)\right\rceil-1,2}
\end{array}\right),
\end{aligned}
$$


the singleton block $\left(v_{\beta}\right)$, as well as $2^{\left\lceil\log _{2}(p)\right\rceil-1}$ blocks of the form

$$
\left(\begin{array}{cc}
u & y_{1, l} \\
y_{1, l} & w
\end{array}\right) \quad \text { for } l \in\left[2^{\left\lceil\log _{2}(p)\right\rceil-1}\right],
$$

where in each of these blocks $u$ and $w$ represent a variable of the set $\left\{v_{\alpha}: \alpha \in A\right\} \cup\left\{v_{\beta}\right\}$ such that altogether each $v_{\alpha}$ appears $p_{\alpha}$ times and $v_{\beta}$ appears $2^{\left\lceil\log _{2}(p)\right\rceil}-p$ times.

In this definition, the exact order of appearances of the variables in $\left\{v_{\alpha}: \alpha \in\right.$ $A\} \cup\left\{v_{\beta}\right\}$ is not uniquely determined. However, since this order of appearances will not matter, we will speak of the dual circuit matrix.

Remark 3.2 Each block of the type (3.4) contains two (not necessarily identical) variables from the set $\left\{v_{\alpha}: \alpha \in A\right\} \cup\left\{v_{\beta}\right\}$. Since $\sum_{\alpha \in A} \lambda_{\alpha}=1$, we have $\sum_{\alpha \in A} p_{\alpha}=p$ and hence the total number of occurrences of variables from the set $\left\{v_{\alpha}: \alpha \in A\right\} \cup\left\{v_{\beta}\right\}$ in the blocks of type (3.4) is

$$
\sum_{\alpha \in A} p_{\alpha}+\left(2^{\left\lceil\log _{2}(p)\right\rceil}-p\right)=2^{\left\lceil\log _{2}(p)\right\rceil},
$$

which is twice the number of blocks of type (3.4).

Note that every $y_{k, i}$ only serves as an auxiliary variable to make the non-linear constraints $\ln \left(v_{\beta}\right) \leq \sum_{\alpha \in A} \lambda_{\alpha} \ln \left(v_{\alpha}\right)$ of the dual $\mathcal{S}$-cone description from Proposition 2.4 linear. In the end, we will only multiply those constraints to obtain the original ones. In particular, factors $v_{\beta}$ serve to cover cases where $p$ is not a power of 2 . For the purpose of the second-order descriptions, it does not matter in which order the variables appear in the blocks (3.4), because only the product of these blocks will be considered.

The goal of this subsection is to show the following characterization of the cone of non-negative even AG functions $P_{A, \beta}^{\text {even }}$ supported on the circuit $(A, \beta)$. Here, positive semidefiniteness of a symmetric matrix is denoted by $\succeq 0$.

Theorem 3.3 The dual cone $\left(P_{A, \beta}^{\mathrm{even}}\right)^{*}$ of the cone of non-negative even AG functions $P_{A, \beta}^{\text {even }}$ supported on the circuit $(A, \beta) \in I(\mathcal{A})$ is the projection of the spectrahedron

$$
\left\{(\mathbf{v}, \mathbf{y}) \in \mathbb{R}^{\mathcal{A}} \times \mathbb{R}^{2^{\left\lceil\log _{2}(p)\right\rceil}-2}: C_{A, \beta}^{*}\left(\mathbf{v}_{\mid A}, v_{\beta}, \mathbf{y}\right) \succcurlyeq 0\right\}
$$

on $\left(\mathbf{v}_{\mid A}, v_{\beta}\right) .\left(P_{A, \beta}^{\text {even }}\right)^{*}$ is second-order representable.

Here, the second-order representability follows immediately from the representation (3.5) in connection with Lemma 2.5. Let us consider an example for the theorem.

Example 3.4 Let $\mathcal{A}=\{0,6\}, \mathcal{B}=\{2\}$ and consider the circuit $(A, \beta)$ with $A=\mathcal{A}$ and $\beta=2$ (compare Fig. 1). We have $p=3, p_{0}=2, p_{6}=1$ and $\mathbf{y}$ consists of the components 
A vector $\left(v_{0}, v_{2}, v_{6}\right)$ is contained in $\left(P_{A, \beta}^{\text {even }}\right)^{*}$ if and only if $v_{2} \geq 0$ and the three $2 \times 2$-matrices

$$
\left(\begin{array}{cc}
y_{1,1} & v_{2} \\
v_{2} & y_{1,2}
\end{array}\right),\left(\begin{array}{cc}
v_{0} & y_{1,1} \\
y_{1,1} & v_{0}
\end{array}\right),\left(\begin{array}{cc}
v_{6} & y_{1,2} \\
y_{1,2} & v_{2}
\end{array}\right)
$$

are positive semidefinite.

In Averkov (2019), Averkov considered the size of the blocks in the SDPrepresentation of SONC-polynomials but does not give a number or bound on the number of blocks. Here, for the $\mathcal{S}$-cone, we provide a bound on the number of inequalities of a second-order representation, which also gives a bound on the number of $2 \times 2$-blocks in a semidefinite representation. The bound depends on the smallest common denominator of the barycentric coordinates representing the inner exponent of a circuit as a convex combination of the outer ones.

Corollary 3.5 The matrix $C_{A, \beta}^{*}\left(\mathbf{v}_{\mid A}, v_{\beta}, \mathbf{y}\right)$ consists of $2^{\left\lceil\log _{2}(p)\right\rceil}-1$ blocks of size $2 \times 2$ and one block of size $1 \times 1$.

Proof Counting the number of $2 \times 2$-blocks, there are $\sum_{k=2}^{\left\lceil\log _{2}(p)\right\rceil-1}\left(2^{\left\lceil\log _{2}(p)\right\rceil-k}\right)=$ $2^{\left\lceil\log _{2}(p)\right\rceil-1}-2$ blocks of type (3.2), a single block (3.3) and $2^{\left\lceil\log _{2}(p)\right\rceil-1}$ blocks of type (3.4).

Remark 3.6 It is useful to record the set inequalities characterizing the positive semidefiniteness of the matrix $C_{A, \beta}^{*}\left(\mathbf{v}_{\mid A}, v_{\beta}, \mathbf{y}\right)$. Besides the non-negativity conditions for the variables,

$$
\begin{aligned}
& \mathbf{v}_{\mid A} \geq 0, \quad v_{\beta} \geq 0, \\
& \quad \text { and } x_{k, i} \geq 0 \text { for all } k \in\left\{2, \ldots,\left\lceil\log _{2}(p)\right\rceil-1\right\}, i \in\left[2^{\left\lceil\log _{2}(p)\right\rceil}-k\right],
\end{aligned}
$$

these are the determinantal conditions arising from the positive semidefiniteness of the matrices in (3.2), (3.3) and (3.4):

$$
\begin{aligned}
v_{\beta}^{2} & \leq y_{\left\lceil\log _{2}(p)\right\rceil-1,1} y_{\left\lceil\log _{2}(p)\right\rceil-1,2}, \\
y_{k, i}^{2} & \leq y_{k-1,2 i-1} y_{k-1,2 i} \text { for all } k \in\left\{2, \ldots,\left\lceil\log _{2}(p)\right\rceil-1\right\}, i \in\left[2^{\left\lceil\log _{2}(p)\right\rceil-k}\right] \\
& \text { and } u w \geq\left(y_{1, l}\right)^{2} \text { for } l \in\left[2^{\left\lceil\log _{2}(p)\right\rceil-1}\right]
\end{aligned}
$$

for $u, w \in\left\{v_{\alpha}: \alpha \in A\right\} \cup\left\{v_{\beta}\right\}$, such that $v_{\alpha}$ appears $p_{\alpha}$ times for every $\alpha \in A$ and $v_{\beta}$ appears $2^{\left\lceil\log _{2}(p)\right\rceil}-p$ times.

The next lemma prepares one inclusion of Theorem 3.3. 
Lemma 3.7 Let $\mathbf{v} \in \mathbb{R}^{A, \beta}$ such that there exists $\mathbf{y} \in \mathbb{R}^{2^{\left\lceil\log _{2}(p)\right\rceil}-2}$ with $C_{A, \beta}^{*}\left(\mathbf{v}_{\mid A}, v_{\beta}, \mathbf{y}\right) \succcurlyeq$ 0 . Then $\mathbf{v}_{\mid A}$ is non-negative and satisfies

$$
v_{\beta}^{p} \leq \prod_{\alpha \in A} v_{\alpha}^{p_{\alpha}}
$$

Proof By (3.6), we have $\mathbf{v}_{\mid A} \geq 0$ and $v_{\beta} \geq 0$. Moreover, (3.8) and successively applying (3.9) gives

$$
\begin{aligned}
& v_{\beta} \leq\left(y_{\left\lceil\log _{2}(p)\right\rceil-1,1} y_{\left\lceil\log _{2}(p)\right\rceil-1,2}\right)^{1 / 2} \\
& \leq\left(y_{\left\lceil\log _{2}(p)\right\rceil-2,1} y_{\left\lceil\log _{2}(p)\right\rceil-2,2}\right)^{1 / 4}\left(y_{\left\lceil\log _{2}(p)\right\rceil-2,3} y_{\left\lceil\log _{2}(p)\right\rceil-2,4}\right)^{1 / 4} \\
& =\left(y_{\left\lceil\log _{2}(p)\right\rceil-2,1} y_{\left\lceil\log _{2}(p)\right\rceil-2,2} y_{\left\lceil\log _{2}(p)\right\rceil-2,3} y_{\left\lceil\log _{2}(p)\right\rceil-2,4}\right)^{\frac{1}{2^{\left\lceil\log _{2}(p)\right\rceil-\left(\left\lceil\log _{2}(p)\right\rceil-2\right)}}} \\
& \leq \cdots \leq\left(\left(\prod_{\alpha \in A} v_{\alpha}^{p_{\alpha}}\right) \cdot\left(v_{\beta}\right)^{2^{\left\lceil\log _{2}(p)\right\rceil}-p}\right)^{\frac{1}{2^{\left[\log _{2}(p)\right\rceil}}} .
\end{aligned}
$$

This is equivalent to

$$
\left(v_{\beta}\right)^{2^{\left\lceil\log _{2}(p)\right\rceil}} \cdot\left(v_{\beta}\right)^{p-2^{\left\lceil\log _{2}(p)\right\rceil}} \leq \prod_{\alpha \in A} v_{\alpha}^{p_{\alpha}}
$$

which implies $v_{\beta}^{p} \leq \prod_{\alpha \in A} v_{\alpha}^{p_{\alpha}}$.

Now we prepare the converse inclusion of Theorem 3.3.

Lemma 3.8 For every $\mathbf{v} \in \mathbb{R}^{A, \beta}$ with $\mathbf{v}_{\mid A \cup\{\beta\}} \geq 0$ and $v_{\beta}^{p} \leq \prod_{\alpha \in A} v_{\alpha}^{p_{\alpha}}$, there exists $\mathbf{y} \in \mathbb{R}^{2^{\left\lceil\log _{2}(p)\right\rceil}-2}$ such that $C_{A, \beta}^{*}\left(\mathbf{v}_{\mid A}, v_{\beta}, \mathbf{y}\right) \succcurlyeq 0$.

Proof Define $\mathbf{y}$ inductively by

$y_{1, l}=\sqrt{u w}$ for those $u, w$ which occur in the block with $y_{1, l}$,

$y_{k, i}=\sqrt{y_{k-1,2 i-1} y_{k-1,2 i}}$ for all $k \in\left\{2, \ldots,\left\lceil\log _{2}(p)\right\rceil-1\right\}, i \in\left[2^{\left\lceil\log _{2}(p)\right\rceil-k}\right]$.

It suffices to show that the inequalities (3.6)-(3.10) in Remark 3.6 are satisfied. The non-negativity conditions (3.6) and (3.7) hold by assumption and by definition of $\mathbf{y}$. The construction of $\mathbf{y}$ also implies that a subchain of the chain of inequalities considered in the previous proof even holds with equality,

$$
\begin{aligned}
& \left(y_{\left\lceil\log _{2}(p)\right\rceil-1,1} y_{\left\lceil\log _{2}(p)\right\rceil-1,2}\right)^{1 / 2} \\
& =\left(y_{\left\lceil\log _{2}(p)\right\rceil-2,1} y_{\left\lceil\log _{2}(p)\right\rceil-2,2}\right)^{1 / 4}\left(y_{\left\lceil\log _{2}(p)\right\rceil-2,3} y_{\left\lceil\log _{2}(p)\right\rceil-2,4}\right)^{1 / 4} \\
& =\left(y_{\left\lceil\log _{2}(p)\right\rceil-2,1} y_{\left\lceil\log _{2}(p)\right\rceil-2,2} y_{\left\lceil\log _{2}(p)\right\rceil-2,3} y_{\left\lceil\log _{2}(p)\right\rceil-2,4}\right)^{\frac{1}{2^{\left\lceil\log _{2}(p)\right\rceil-\left(\left\lceil\log _{2}(p)\right\rceil-2\right)}}} \\
& =\cdots=\left(\left(\prod_{\alpha \in A} v_{\alpha}^{p_{\alpha}}\right) \cdot\left(v_{\beta}\right)^{2^{\left[\log _{2}(p)\right\rceil}-p}\right)^{\frac{1}{2^{\left[\log _{2}(p)\right\rceil}}} .
\end{aligned}
$$


By the assumption $v_{\beta}^{p} \leq \prod_{\alpha \in A} v_{\alpha}^{p_{\alpha}}$, we obtain $v_{\beta}^{2} \leq y_{\left\lceil\log _{2}(p)\right\rceil-1,1} y_{\left\lceil\log _{2}(p)\right\rceil-1,2}$, which shows inequality (3.8). The remaining inequalities (3.9), (3.10) are satisfied with equality by construction.

Finally, we can conclude the proof of Theorem 3.3.

Proof of Theorem 3.3 Let $p$ be defined as in Definition 3.1 and $\lambda \in \mathbb{R}^{A}$ denote the barycentric coordinates representing $\beta$ as a convex combination of $A$, i.e., $\lambda_{\alpha}=\frac{p_{\alpha}}{p}$ with $p_{\alpha} \in \mathbb{N}$ for all $\alpha \in A$. By (2.3) and Proposition 2.4, we have

$$
\begin{aligned}
\left(P_{A, \beta}^{\text {even }}\right)^{*} & =\left\{\mathbf{v} \in \mathbb{R}^{A, \beta}: \mathbf{v}_{\mid A \cup\{\beta\}} \geq 0, \ln \left(v_{\beta}\right) \leq \sum_{\alpha \in A} \lambda_{\alpha} \ln \left(v_{\alpha}\right)\right\} \\
& =\left\{\mathbf{v} \in \mathbb{R}^{A, \beta}: \mathbf{v}_{\mid A \cup\{\beta\}} \geq 0, v_{\beta}^{p} \leq \prod_{\alpha \in A} v_{\alpha}^{p_{\alpha}}\right\} .
\end{aligned}
$$

Applying Lemmas 3.7 and 3.8, we obtain that $C_{A, \beta}^{*}\left(x, v_{\beta}\right) \succcurlyeq 0$ if and only if $\mathbf{v} \in P_{A, \beta}^{*}$.

Our derivation of the second-order representation of the dual cone $\left(P_{A, \beta}^{\text {even }}\right)^{*}$ also suggests a simple way to derive a second-order cone representation of the primal cone $P_{A, \beta}^{\text {even }}$. For the dual cone, Proposition 2.4 gives-besides non-negativity-constraints on $v_{\alpha}$ for $\alpha \in \mathcal{A}$ and on $v_{\beta}$-the condition $\ln \left(v_{\beta}\right) \leq \sum_{\alpha \in A} \lambda_{\alpha} \ln \left(v_{\alpha}\right)$ for every circuit $(A, \beta) \in I(\mathcal{A})$. Those conditions can-as done in the previous proof - be stated as

$$
v_{\beta}^{p} \leq \prod_{\alpha \in A} v_{\alpha}^{p_{\alpha}}, \text { where } \lambda_{\alpha}=\frac{p_{\alpha}}{p}
$$

The conditions for the primal cone can be reformulated similarly. Namely, by (2.6), an even circuit function $f$ with coefficient vector $\mathbf{c}$ is non-negative if and only if $-c_{\beta} \leq \prod_{\alpha \in A}\left(c_{\alpha} / \lambda_{\alpha}\right)^{\lambda_{\alpha}}$, which we write as

$$
\left(-c_{\beta}\right)^{p} \leq \prod_{\alpha \in A}\left(\frac{c_{\alpha}}{\lambda_{\alpha}}\right)^{p_{\alpha}}
$$

This motivates to carry over the definition of the dual circuit matrix to the primal case as follows. Since $c_{\beta}$ may be negative (in contrast to the dual case), we introduce the primal circuit variables, or simply circuit variables,

$$
\left(x_{\beta},\left(x_{k, i}\right)_{k, i}\right)
$$

where $k \in\left[\left\lceil\log _{2}(p)\right\rceil\right]$ and $i \in\left[2^{\left\lceil\log _{2}(p)\right\rceil-k}\right]$. As in the dual case, we refer to these $1+\sum_{k=1}^{\left\lceil\log _{2}(p)\right\rceil} 2^{\left\lceil\log _{2}(p)\right\rceil-k}=2^{\left\lceil\log _{2}(p)\right\rceil}$ variables as $\mathbf{x}^{A, \beta}$ or shortly as $\mathbf{x}$.

Definition 3.9 (Circuit matrix) The circuit matrix $C_{A, \beta}\left(\mathbf{c}_{\mid A \cup\{\beta\}}, x_{\beta}, \mathbf{x}\right.$ ) is the block diagonal matrix consisting of the blocks

$$
\left(\begin{array}{cc}
x_{k-1,2 i-1} & x_{k, i} \\
x_{k, i} & x_{k-1,2 i}
\end{array}\right) \quad \text { for } k \in\left\{2, \ldots,\left\lceil\log _{2}(p)\right\rceil\right\}, i \in\left[2^{\left\lceil\log _{2}(p)\right\rceil-k}\right] \text {, }
$$


the two singleton blocks

$$
\left(x_{\left\lceil\log _{2}(p)\right\rceil, 1}-\left(\prod_{\alpha \in A}\left(\lambda_{\alpha}\right)^{\lambda_{\alpha}}\right) x_{\beta}\right), \quad\left(x_{\beta}+c_{\beta}\right),
$$

as well as $2^{\left\lceil\log _{2}(p)\right\rceil-1}$ blocks of the form

$$
\left(\begin{array}{cc}
u & x_{1, l} \\
x_{1, l} & w
\end{array}\right) \quad \text { for } l \in\left[2^{\left\lceil\log _{2}(p)\right\rceil-1}\right]
$$

where $u, w \in\left\{c_{\alpha}: \alpha \in A\right\} \cup\left\{\left(\prod_{\alpha \in A}\left(\lambda_{\alpha}\right)^{\lambda_{\alpha}}\right) x_{\beta}\right\}$, such that $c_{\alpha}$ appears $p_{\alpha}$ times for every $\alpha \in A$ and $\left(\prod_{\alpha \in A}\left(\lambda_{\alpha}\right)^{\lambda_{\alpha}}\right) x_{\beta}$ appears $2^{\left\lceil\log _{2}(p)\right\rceil}-p$ times.

Note that for a circuit $(A, \beta)$, the product $\left(\prod_{\alpha \in A}\left(\lambda_{\alpha}\right)^{\lambda_{\alpha}}\right)$ is always non-zero, because $\beta \in$ relint conv $A$ and $A$ consists of affinely independent vectors.

In contrast to the dual cone, there is no sign constraint on $c_{\beta}$ in the primal cone. If $p$ is not a power of 2 , then $x_{\beta}$ appears on the main diagonal of (3.12). In our coupling of $x_{\beta}$ with $c_{\beta}$, the constraint $x_{\beta}+c_{\beta} \geq 0$ results in $-c_{\beta} \leq x_{\beta}$ and thus reflects these sign considerations.

Note that the primal cone consists of circuit functions, whereas in our definition of the dual cone, the elements are coefficient vectors. Therefore, the projection regarded in Theorem 3.3 only delivers the coefficients of the circuit functions rather than the cone itself.

Theorem 3.10 The set of coefficients of the cone $P_{A, \beta}^{\mathrm{even}}$ of non-negative even circuit polynomials supported on the circuit $(A, \beta)$ coincides with the projection of the spectrahedron

$$
\widehat{P_{A, \beta}^{\text {even }}}:=\left\{(\mathbf{c}, \mathbf{x}) \in \mathbb{R}^{\mathcal{A}} \times \mathbb{R}^{2^{\left\lceil\log _{2}(p)\right\rceil}}: C_{A, \beta}\left(\mathbf{c}_{\mid A \cup\{\beta\}}, x_{\beta}, \mathbf{x}\right) \succcurlyeq 0, c_{\mid \mathcal{A} \backslash(A \cup\{\beta\})}=0\right\}
$$

on $\left(\mathbf{c}_{\mid A}, c_{\beta}\right)$. The cone $P_{A, \beta}^{\mathrm{even}}$ is second-order representable.

The last equality constraint in (3.13) is redundant and can be omitted. We include it here, because this formulation is needed in Sect. 4 for the description of the $\mathcal{S}$-cone supported on the full set $\mathcal{A}$.

Proof First, let $(\mathbf{c}, \mathbf{x}) \in \widehat{P_{A, \beta}^{\text {even }}}$. The positive semidefiniteness of the $2 \times 2$-blocks in $C_{A, \beta}\left(\mathbf{c}_{\mid A \cup\{\beta\}}, x_{\beta}, \mathbf{x}\right)$ imply the inequalities

$$
\mathbf{c}_{\mid A} \geq 0 \text { and }\left(-x_{\beta}\right)^{p} \cdot\left(\prod_{\alpha \in A} \lambda_{\alpha}^{\lambda_{\alpha}}\right) \leq \prod_{\alpha \in A} c_{\alpha}^{p_{\alpha}}
$$

The two $1 \times 1$-blocks from (3.11) give the inequalities $x_{\left\lceil\log _{2}(p)\right\rceil, 1} \geq\left(\prod_{\alpha \in A} \lambda_{\alpha}^{\lambda_{\alpha}}\right) x_{\beta}$ and $x_{\beta} \geq-c_{\beta}$. They imply $-c_{\beta}\left(\prod_{\alpha \in A} \lambda_{\alpha}^{\lambda_{\alpha}}\right) \leq x_{\beta}\left(\prod_{\alpha \in A} \lambda_{\alpha}^{\lambda_{\alpha}}\right) \leq x_{\left\lceil\log _{2}(p)\right\rceil, 1}$. Hence, similar to Lemma 3.7,

$$
x_{\beta}\left(\prod_{\alpha \in A} \lambda_{\alpha}^{\lambda_{\alpha}}\right) \leq x_{\left\lceil\log _{2}(p)\right\rceil, 1} \leq\left(x_{\left\lceil\log _{2}(p)\right\rceil-1,1} x_{\left\lceil\log _{2}(p)\right\rceil-1,2}\right)^{1 / 2}
$$




$$
\begin{aligned}
& \leq\left(x_{\left\lceil\log _{2}(p)\right\rceil-2,1} x_{\left\lceil\log _{2}(p)\right\rceil-2,2}\right)^{1 / 4}\left(x_{\left\lceil\log _{2}(p)\right\rceil-2,3} x_{\left\lceil\log _{2}(p)\right\rceil-2,4}\right)^{1 / 4} \\
& =\left(x_{\left\lceil\log _{2}(p)\right\rceil-2,1} x_{\left\lceil\log _{2}(p)\right\rceil-2,2} x_{\left\lceil\log _{2}(p)\right\rceil-2,3} x_{\left\lceil\log _{2}(p)\right\rceil-2,4}\right)^{\frac{2^{\left\lceil\log _{2}(p)\right\rceil-\left(\left\lceil\log _{2}(p)\right\rceil-2\right)}}{}} \\
& \leq \cdots \leq\left(\left(\prod_{\alpha \in A} c_{\alpha}^{p_{\alpha}}\right) \cdot\left(x_{\beta}\right)^{2^{\left\lceil\log _{2}(p)\right\rceil}-p}\left(\prod_{\alpha \in A} \lambda_{\alpha}^{\lambda_{\alpha}}\right)^{2^{\left\lceil\log _{2}(p)\right\rceil}-p}\right)^{\frac{1}{2^{\left\lceil\log _{2}(p)\right\rceil}}}
\end{aligned}
$$

This is equivalent to

$$
\begin{aligned}
\left(x_{\beta}\right)^{2^{\left\lceil\log _{2}(p)\right\rceil}} \cdot\left(\prod_{\alpha \in A} \lambda_{\alpha}^{\lambda_{\alpha}}\right)^{2^{\left\lceil\log _{2}(p)\right\rceil}} \cdot\left(x_{\beta}\right)^{p-2^{\left\lceil\log _{2}(p)\right\rceil}} & \leq \prod_{\alpha \in A} c_{\alpha}^{p_{\alpha}},
\end{aligned}
$$

which, together with the considerations before the chain of inequalities, yields $\left(-c_{\beta}\right)^{p} \leq \prod_{\alpha \in A}\left(c_{\alpha} / \lambda_{\alpha}\right)^{p_{\alpha}}$ and further $\mathbf{c}_{\mid A \cup\{\beta\}} \in P_{A, \beta}^{\text {even }}$.

For the converse inclusion, we remind the reader that $\lambda_{\alpha}>0$ for all $\alpha \in A$. We set $x_{\beta}:=x_{\left\lceil\log _{2}(p)\right\rceil, 1}\left(\prod_{\alpha \in A}\left(\frac{1}{\lambda_{\alpha}}\right)^{\lambda_{\alpha}}\right)$ and, similar to the proof of Lemma 3.8, define $\mathbf{x}$ inductively by

$x_{1, l}=\sqrt{u w}$ for those $u, w$ which occur in the block with $x_{1, l}$,

$x_{k, i}=\sqrt{x_{k-1,2 i-1} x_{k-1,2 i}}$ for all $k \in\left\{2, \ldots,\left\lceil\log _{2}(p)\right\rceil\right\}, i \in\left[2^{\left\lceil\log _{2}(p)\right\rceil-k}\right]$.

Analogous to that proof, the construction of $\mathbf{x}$ gives $C_{A, \beta}\left(\mathbf{c}_{A \cup\{\beta\}}, x_{\beta}, \mathbf{x}\right) \succeq 0$.

Second-order representability is then an immediate consequence in view of Lemma 2.5.

Example 3.11 Let $\mathcal{A}=\{0,2\}, \mathcal{B}=\{1\}$ and consider the circuit $(A, \beta)$ with $A=\mathcal{A}$ and $\beta=1$. Since

$$
1=\frac{1}{2} \cdot 0+\frac{1}{2} \cdot 2
$$

we have $p_{1}=p_{2}=1$ and $p=2$. Hence, $\left\lceil\log _{2}(p)\right\rceil=\log _{2}(p)=1,2^{\left\lceil\log _{2}(p)\right\rceil}-p=$ $2-p=0$ as well as

$$
\prod_{\alpha \in A} \lambda_{\alpha}^{\lambda_{\alpha}}=\frac{1}{2} \text { and } \mathbf{x}=\left(\begin{array}{c}
x_{1} \\
x_{1,1}
\end{array}\right)
$$

A given vector $\left(c_{0}, c_{1}, c_{2}\right)$ is contained in $P_{\mathcal{A}, \beta}$ if and only if

$$
x_{1,1}-\frac{1}{2} x_{1} \geq 0, x_{1}+c_{1} \geq 0 \text { and }\left(\begin{array}{cc}
c_{0} & x_{1,1} \\
x_{1,1} & c_{2}
\end{array}\right) \succeq 0 \text {. }
$$

Similar to Lemma 3.5, we can determine the number of blocks. 
Corollary 3.12 The matrix $C_{A, \beta}\left(\mathbf{c}_{\mid A \cup\{\beta\}}, x_{\beta}, \mathbf{x}\right)$ consists of $2^{\left\lceil\log _{2}(p)\right\rceil}-1$ blocks of size $2 \times 2$ and two blocks of size $1 \times 1$.

\section{A second-order representation of the $\mathcal{S}$-cone and its dual}

In Sect. 3, we obtained second-order representations of the subcones of non-negative even circuit functions and their duals, under the condition that the barycentric coordinates are rational. We now assume that $\mathcal{A}$ and $\mathcal{B}$ are rational and derive an explicit second-order representation of the rational $\mathcal{S}$-cone $C_{\mathcal{S}}(\mathcal{A}, \mathcal{B})$ and its dual. In the primal case, those cones are obtained via projection and Minkowski sum, and in the dual case, they arise from projection and intersection. First we consider the lifted cones for the dual case.

Taking all circuits $(A, \beta)$ into account would induce a highly redundant representation. To avoid those redundancies, we make use of the following characterization from Katthän et al. (2019) of the extreme rays of the $\mathcal{S}$-cone.

For finite and disjoint sets $\emptyset \neq \mathcal{A}, \mathcal{B} \subseteq \mathbb{R}^{n}$, the set of reduced circuits contained in $\mathcal{A} \cup \mathcal{B}$ is the set

$$
\begin{aligned}
R(\mathcal{A}, \mathcal{B})=\{ & (A, \beta): A \subseteq \mathcal{A} \text { affinely independent, } \quad \beta \in \operatorname{relint}(\operatorname{conv} A) \cap(\mathcal{B} \backslash A), \\
& \mathcal{A} \cap(\operatorname{conv}(A)) \backslash(A \cup\{\beta\})=\emptyset\} .
\end{aligned}
$$

Less formally, this is the set of all circuits with outer exponents in $\mathcal{A}$ and inner exponents in $\mathcal{B}$ without additional support points contained in the convex hull of the circuit.

Note that for $\mathcal{A} \subseteq \mathbb{R}^{n}$ and $\mathcal{B} \subseteq \mathbb{N}^{n} \backslash(2 \mathbb{N})^{n}$ disjoint and finite, the set $R(\mathcal{A}, \mathcal{A})$ is exactly the set of even reduced circuits and the set $R(\mathcal{A}, \mathcal{B})$ the set of odd reduced circuits. The set $R(\mathcal{A}, \mathcal{A} \cup \mathcal{B})$ denotes the set of all reduced circuits $(A, \beta)$ with $A \subseteq \mathcal{A}$ and $\beta \in \mathcal{A} \cup \mathcal{B}$. A circuit function supported on a reduced circuit in $R(\mathcal{A}, \mathcal{A} \cup \mathcal{B})$ has non-negative coefficients corresponding to exponents in $\mathcal{A}$ and a possibly negative coefficient corresponding to a single exponent in $\mathcal{A} \cup \mathcal{B}$.

The question whether a circuit is reduced or not depends on the ground set $\mathcal{A}$. For example, the circuit $(A, \beta)$ with $A=\left\{\left(\begin{array}{l}0 \\ 0\end{array}\right),\left(\begin{array}{l}4 \\ 0\end{array}\right),\left(\begin{array}{l}0 \\ 2\end{array}\right)\right\}$ and $\beta=\left(\begin{array}{l}1 \\ 1\end{array}\right)$ is reduced for the ground set $\mathcal{A}=A \cup\{\beta\} \cup\left\{\left(\begin{array}{l}4 \\ 2\end{array}\right)\right\}$ (compare Fig. 3), but not reduced for $\mathcal{A}=A \cup\{\beta\} \cup\left\{\left(\begin{array}{l}2 \\ 0\end{array}\right)\right\}$ (compare Fig. 4).

The following proposition is a direct consequence of Theorem 3.5(d) in Katthän et al. (2019).

Proposition 4.1 Let $\emptyset \neq \mathcal{A} \subseteq \mathbb{R}^{n}$ and $\mathcal{B} \subseteq \mathbb{N}^{n} \backslash(2 \mathbb{N})^{n}$ be finite and disjoint sets. Then

$$
C_{\mathcal{S}}(\mathcal{A}, \mathcal{B})=\sum_{(A, \beta) \in R(\mathcal{A}, \mathcal{A})} P_{A, \beta}^{\text {even }}+\sum_{(A, \beta) \in R(\mathcal{A}, \mathcal{B})} P_{A, \beta}^{\text {odd }}
$$


Fig. 3 The circuit is reduced, as $(4,2)^{T} \notin \operatorname{conv}(A)$

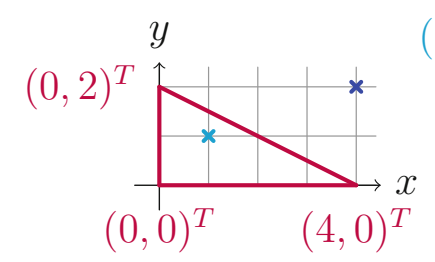

$(1,1)^{T} \quad(4,2)^{T}$

Fig. 4 The circuit is not reduced, as $(2,0)^{T} \in \operatorname{conv}(A)$

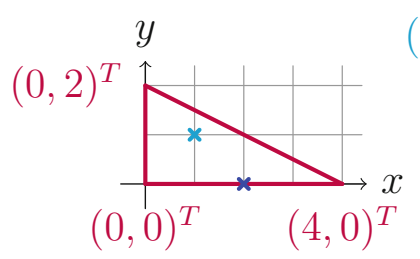

$(1,1)^{T} \quad(2,0)^{T}$

Using this decomposition theorem, we can exclude many circuits from our consideration. Thus, the second-order program will be much smaller than the one considering all circuits.

In Sect. 3, we only considered even circuits. To use Lemma 2.1 and obtain the conditions for odd circuits as well, we extend the dual circuit variables for odd circuits to

$$
\left(y_{\beta},\left(y_{k, i}\right)_{k, i}\right)
$$

for $k \in\left[2^{\left\lceil\log _{2}(p)\right\rceil}-1\right]$ and $i \in\left[2^{\log _{2}(p)-k}\right]$. We call them $\mathbf{y}^{A, \beta}$ nevertheless for a fixed circuit $(A, \beta) \in R(\mathcal{A}, \mathcal{B})$.

For the dual case, we consider the coordinates

$$
\mathbf{y}^{\mathcal{A}, \mathcal{B}}=\left\{\left(\mathbf{y}^{A, \beta}\right):(A, \beta) \in R(\mathcal{A}, \mathcal{A} \cup \mathcal{B})\right\}
$$

which consist of $\sum_{(A, \beta) \in R(\mathcal{A}, \mathcal{A} \cup \mathcal{B})} 2^{\left\lceil\log _{2}\left(p_{A, \beta}\right)\right\rceil}-1$ components, where $p_{A, \beta}$ denotes the smallest common denominator of the barycentric coordinates $\lambda_{A, \beta}$ of the circuit $(A, \beta)$ representing $\beta$ as a convex combination of $A$.

For the primal case, we consider

$$
\mathbf{x}^{\mathcal{A}, \mathcal{B}}=\left\{\left(\mathbf{x}^{A, \beta}\right):(A, \beta) \in R(\mathcal{A}, \mathcal{A} \cup \mathcal{B})\right\}
$$

which consist of $\sum_{(A, \beta) \in R(\mathcal{A}, \mathcal{A} \cup \mathcal{B})} 2^{\left\lceil\log _{2}\left(p_{A, \beta}\right)\right\rceil}$ components.

Using Lemma 2.1, we can use our earlier characterizations of $P_{A, \beta}^{\text {even }}$ to obtain the following second-order characterization for $P_{A, \beta}^{\text {odd }}$.

Corollary 4.2 Let $(A, \beta) \in R(\mathcal{A}, \mathcal{B})$ an odd reduced circuit with rational $A \subseteq \mathcal{A} \subseteq \mathbb{Q}^{n}$ and $\beta \in \mathcal{B}$. 
1. Let $f$ be an odd AG function supported on $(A, \beta)$ with coefficient vector $\mathbf{c} f$ is nonnegative if and only if there exists $\mathbf{x} \in \mathbb{R}^{2^{\left\lceil\log _{2}(p)\right\rceil}}$ such that $C_{A, \beta}\left(\mathbf{c}_{\mid A}, x_{\beta}, \mathbf{x}\right) \succcurlyeq 0$ and

$$
\left(\begin{array}{ll}
x_{\beta} & c_{\beta} \\
c_{\beta} & x_{\beta}
\end{array}\right) \succcurlyeq 0
$$

2. A vector $\mathbf{v} \in \mathbb{R}^{A, \beta}$ is contained in $\left(P_{A, \beta}^{\text {odd }}\right)^{*}$ if and only if there exist $\mathbf{y} \in \mathbb{R}^{2^{\left[\log _{2}(p)\right\rceil}-2}$ and $y_{\beta} \in \mathbb{R}$ such that $C_{A, \beta}^{*}\left(\mathbf{v}_{\mid A}, y_{\beta}, \mathbf{y}\right) \succcurlyeq 0$ and

$$
\left(\begin{array}{ll}
y_{\beta} & v_{\beta} \\
v_{\beta} & y_{\beta}
\end{array}\right) \succcurlyeq 0
$$

Note that, as a consequence of the application of Lemma 2.1, the second argument of $C_{A, \beta}^{*}\left(\mathbf{v}_{\mid A}, y_{\beta}, \mathbf{y}\right)$ is $y_{\beta}$ now instead of $v_{\beta}$ that we had in Theorem 3.3.

Proof 1. The semidefinite condition on the matrix (4.1) is equivalent to $x_{\beta} \geq$ 0 and $\left|c_{\beta}\right| \leq x_{\beta}$. Hence, altogether we obtain

$$
f \in P_{A, \beta}^{\text {odd }} \text { if and only if }\left|c_{\beta}\right| \leq \prod_{\alpha \in A}\left(\frac{c_{\alpha}}{\lambda}\right)^{\lambda_{\alpha}}
$$

for barycentric coordinates $\lambda \in \mathbb{R}_{+}^{A}$ decomposing $\beta$ as a convex combination of $A$. This is exactly Proposition 2.2(b).

2. If $\mathbf{v} \in\left(P_{A, \beta}^{\text {odd }}\right)^{*}$, then, in the notation of Theorem 2.9, there exists some $u$ such that $(\mathbf{v}, u) \in\left(P_{A, \beta}^{\text {even }}\right)^{*}$ and $u \geq\left|v_{\beta}\right|$. In particular, $u \geq 0$ is necessary for containment in $\left(P_{A, \beta}^{\text {even }}\right)^{*}$. The semidefinite constraints (4.2) are equivalent to $y_{\beta} \geq 0$ and the latter inequality $u \geq\left|v_{\beta}\right|$, and the constraint $C_{A, \beta}^{*}\left(\mathbf{v}_{\mid A}, y_{\beta}, \mathbf{y}\right) \succcurlyeq 0$ is equivalent to $\left(\mathbf{v}, y_{\beta}\right) \in\left(P_{A, \beta}^{\text {even }}\right)^{*}$ by Theorem 3.3.

For every odd reduced circuit $(A, \beta) \in R(\mathcal{A}, \mathcal{B})$, define the block diagonal matrix $\widehat{C}_{A, \beta}^{*}\left(\mathbf{v}_{\mid A \cup\{\beta\}}, y_{\beta}, \mathbf{y}\right)$ consisting of the dual circuit matrix $C_{A, \beta}^{*}\left(\mathbf{v}_{\mid A \cup\{\beta\}}, y_{\beta}, \mathbf{y}\right)$ and (4.1) for the dual cone. Considering all the reduced circuits, these lifting matrices define the lifted cone

$$
\begin{gathered}
\widehat{C}^{*}(\mathcal{A}, \mathcal{B})=\left\{\left(\mathbf{v}, \mathbf{y}^{\mathcal{A}, \mathcal{B}}\right): \widehat{C}_{A, \beta}^{*}\left(\mathbf{v}_{\mid A \cup\{\beta\}}, y_{\beta}, \mathbf{y}\right) \succcurlyeq 0 \text { for all }(A, \beta) \in R(\mathcal{A}, \mathcal{B}),\right. \\
\left.C_{A, \beta}^{*}\left(\mathbf{v}_{\mid A}, v_{\beta}, \mathbf{y}\right) \succcurlyeq 0 \text { for all }(A, \beta) \in R(\mathcal{A}, \mathcal{A})\right\},
\end{gathered}
$$

where the variable vector $\mathbf{v}$ lives in the space $\mathbb{R}^{\mathcal{A}, \mathcal{B}}$.

For a fixed odd reduced circuit $(A, \beta) \in R(\mathcal{A}, \mathcal{B})$, let

$$
\widehat{P_{A, \beta}^{\text {odd }}}=\left\{\left(\mathbf{c}, \mathbf{x}^{\mathcal{A}, \mathcal{B}}\right): \widehat{C}_{A, \beta}\left(\mathbf{c}_{\mid A \cup\{\beta\}}, x_{\beta}, \mathbf{x}^{A, \beta}\right) \succcurlyeq 0, c_{\mid \mathcal{A} \cup \mathcal{B} \backslash(A \cup\{\beta\})}=0\right\},
$$


where $\widehat{C}_{A, \beta}\left(\mathbf{c}_{\mid A \cup\{\beta\}}, x_{\beta}, \mathbf{x}^{A, \beta}\right)$ is defined analogous to the dual case. We define the lifted cone

$$
\widehat{C}(\mathcal{A}, \mathcal{B})=\sum_{(A, \beta) \in R(\mathcal{A}, \mathcal{A})} \widehat{P_{A, \beta}^{\text {even }}}+\sum_{(A, \beta) \in R(\mathcal{A}, \mathcal{B})} \widehat{P_{A, \beta}^{\text {odd }}}
$$

Here, for every $(A, \beta) \in R(\mathcal{A}, \mathcal{A}), \widehat{P_{A, \beta}^{\mathrm{even}}}$ is the set from Theorem 3.10.

Corollary 4.3 1. The dual of the rational $\mathcal{S}$-cone $C_{\mathcal{S}}^{*}(\mathcal{A}, \mathcal{B})$ is the projection on the coordinates $\mathbf{v} \in \mathbb{R}^{\mathcal{A}, \mathcal{B}}$ of $\widehat{C}^{*}(\mathcal{A}, \mathcal{B})$.

2. The primal rational $\mathcal{S}$-cone $C_{\mathcal{S}}(\mathcal{A}, \mathcal{B})$ is the projection on the coordinates $\mathbf{v} \in$ $\mathbb{R}^{\mathcal{A}, \mathcal{B}}$ of $\widehat{C}(\mathcal{A}, \mathcal{B})$.

Applying this lifting to the second-order representations of Theorems 3.10 and 3.3 in standard form also gives second-order representations of $C_{\mathcal{S}}(\mathcal{A}, \mathcal{B})$ and $C_{\mathcal{S}}^{*}(\mathcal{A}, \mathcal{B})$ in standard form.

Corollary 4.4 (Second-order representation of the dual rational $\mathcal{S}$-cone) A vector $\mathbf{v} \in$ $\mathbb{R}^{(\mathcal{A}, \mathcal{B})}$ is contained in the rational $\mathcal{S}$-cone $\left(C_{\mathcal{S}}(\mathcal{A}, \mathcal{B})\right)^{*}$ if and only if the circuit vector $\mathbf{y}^{\mathcal{A}, \mathcal{B}}$ satisfies for every reduced odd circuit $(A, \beta) \in R(\mathcal{A}, \mathcal{B})$

1. $\left(\begin{array}{cc}y_{k-1,2 i-1}^{A, \beta} & y_{k, i}^{A, \beta} \\ y_{k, i}^{A, \beta} & y_{k-1,2 i}^{A, \beta}\end{array}\right) \succeq 0, \quad 2 \leq k \leq\left\lceil\log _{2}\left(p_{A, \beta}\right)\right\rceil-1 \forall i \in\left[2^{\left\lceil\log _{2}\left(p_{A, \beta}\right)\right\rceil-k}\right]$,

2. $\left(\begin{array}{cc}y_{\left\lceil\log _{2}\left(p_{A, \beta}\right)\right\rceil-1,1}^{A, \beta} & y_{\beta}^{A, \beta} \\ y_{\beta}^{A, \beta} & y_{\left\lceil\log _{2}\left(p_{A, \beta}\right)\right\rceil-1,2}^{A, \beta}\end{array}\right) \succeq 0$,

3. $\left(\begin{array}{cc}u & y_{1, l}^{A, \beta} \\ y_{1, l}^{A, \beta} & w\end{array}\right) \succeq 0$ for $l \in\left[2^{\left\lceil\log _{2}\left(p_{A, \beta}\right)\right\rceil-1}\right]$ and $u, w \in\left\{v_{\alpha}: \alpha \in A\right\} \cup\left\{y_{\beta}^{A, \beta}\right\}$, such that $v_{\alpha}$ appears $\left(p_{A, \beta}\right)_{\alpha}$ times for each $\alpha \in A$ and $y_{\beta}^{A, \beta}$ appears $2^{\left\lceil\log _{2}\left(p_{A, \beta}\right){ }_{-}\right.}$ $\left(p_{A, \beta}\right)_{\alpha}$ times,

4. $\left\|v_{\beta}\right\|_{2} \leq y_{\beta}^{A, \beta}$,

and for every reduced even circuit $(A, \beta) \in R(\mathcal{A}, \mathcal{A})$ the conditions of Theorem 3.3.

We need to write $\mathbf{y}^{A, \beta}$ instead of just writing $\mathbf{y}$ in the previous corollary, since different $\mathbf{y}^{A, \beta}$ for every reduced circuit $(A, \beta)$ may appear.

For the primal case, we have to consider every reduced circuit as well. Here, sums take the role of the intersections from the dual case.

Corollary 4.5 (A second-order representation of the rational $\mathcal{S}$-cone) A function $f \in$ $\mathbb{R}[\mathcal{A}, \mathcal{B}]$ with coefficient vector $\mathbf{c}$ is contained in the rational $\mathcal{S}$-cone $C_{\mathcal{S}}(\mathcal{A}, \mathcal{B})$ if and only if there exists $\mathbf{c}^{A, \beta}$ for $(A, \beta) \in R(\mathcal{A}, \mathcal{A} \cup \mathcal{B})$ with $\mathbf{c}=\sum_{(A, \beta) \in R(\mathcal{A}, \mathcal{A} \cup \mathcal{B})} \mathbf{c}^{A, \beta}$ and for the circuit vector $\mathbf{x}^{\mathcal{A}, \mathcal{B}}$ and for every $(A, \beta) \in R(\mathcal{A}, \mathcal{A} \cup \mathcal{B})$ the following inequalities hold.

1. $\left(\begin{array}{cc}x_{k-1,2 i-1}^{A, \beta} & x_{k, i}^{A, \beta} \\ x_{k, i}^{A, \beta} & x_{k-1,2 i}^{A, \beta}\end{array}\right) \succcurlyeq 0,2 \leq k \leq\left\lceil\log _{2}\left(p_{A, \beta}\right)\right\rceil, i \in\left[2^{\left\lceil\log _{2}\left(p_{A, \beta}\right)\right\rceil-k}\right]$, 
2. $x_{\left\lceil\log _{2}\left(p_{A, \beta}\right)\right\rceil, 1}^{A, \beta}-\left(\prod_{\alpha \in A} \lambda_{\alpha}^{(p A, \beta)_{\alpha}}\right) x_{\beta}^{A, \beta} \geq 0$,

3. $x_{\beta}^{A, \beta}+c_{\beta} \geq 0$,

4. $\left\|c_{\beta}\right\|_{2} \leq x_{\beta}^{A, \beta}$ if $(A, \beta)$ is an odd circuit,

5. as well as in both the even and the odd case,

$$
\left(\begin{array}{cc}
u & x_{1, l}^{A, \beta} \\
x_{1, l}^{A, \beta} & w
\end{array}\right) \succcurlyeq 0 \quad \text { for } l \in\left[2^{\left\lceil\log _{2}\left(\lambda_{A, \beta}\right)\right\rceil-1}\right]
$$

for $u, w \in\left\{c_{\alpha}: \alpha \in A\right\} \cup\left\{\left(\prod_{\alpha \in A} \lambda_{\alpha}^{\left(\lambda_{A, \beta}\right)_{\alpha}}\right) x_{\beta}^{A, \beta}\right\}$, such that $c_{\alpha}$ appears $\left(p_{A, \beta}\right)_{\alpha}$ times for every $\alpha \in A$ and $\left(\prod_{\alpha \in A} \lambda_{\alpha}^{\left(\lambda_{A, \beta}\right)_{\alpha}}\right) x_{\beta}^{A, \beta}$ appears $2^{\left\lceil\log _{2}\left(p_{A, \beta}\right)\right\rceil}-p_{A, \beta}$ times.

As already mentioned in Sect. 2 , the $\operatorname{SONC}$ cone $C_{\mathrm{SONC}}(\mathcal{A})$ and its dual are always rational $\mathcal{S}$-cones and thus occur as a special case of Corollaries 4.5 and 4.4.

Remark 4.6 The specific case of the primal SONC cone has also been studied in detail by Wang and Magron (2019). Their approach is based on different methods. In particular, it relies on mediated sets and intermediately uses sums of squares representations. However, the resulting second-order programs are structurally similar. Notably, the dependence of the size of the second-order program on the parameter $p$ in our derivation relates to the dependency on the size of the rational mediated set in Wang and Magron (2019). Note also that various amendments are integrated into the approaches (such as the handling of denominators in Wang and Magron (2019) and the use of extreme rays in our approach).

\section{Conclusion and open question}

We have provided second-order representations for primal and dual rational $\mathcal{S}$-cones. These statements remain valid also for non-rational sets $\mathcal{A}$, as long as all the relevant barycentric coordinates are still rational. It is an open question whether an $\mathcal{S}$-cone and its dual are also second-order representable in the general non-rational case.

Also, despite the use of the reduced circuits, the second-order representation of the $\mathcal{S}$-cone is still rather large. It remains the question whether smaller second-order representations for the $\mathcal{S}$-cone exist.

Acknowledgements Open Access funding provided by Projekt DEAL. The work was partially supported through the project "Real Algebraic Geometry and Optimization" jointly funded by the German Academic Exchange Service DAAD and the Research Council of Norway RCN. We thank an anonymous referee for some beneficial suggestions.

Open Access This article is licensed under a Creative Commons Attribution 4.0 International License, which permits use, sharing, adaptation, distribution and reproduction in any medium or format, as long as you give appropriate credit to the original author(s) and the source, provide a link to the Creative Commons licence, and indicate if changes were made. The images or other third party material in this article are included in the article's Creative Commons licence, unless indicated otherwise in a credit line to the material. If material is not included in the article's Creative Commons licence and your intended use is not permitted 
by statutory regulation or exceeds the permitted use, you will need to obtain permission directly from the copyright holder. To view a copy of this licence, visit http://creativecommons.org/licenses/by/4.0/.

\section{References}

Averkov, G.: Optimal size of linear matrix inequalities in semidefinite approaches to polynomial optimization. SIAM J. Appl. Algebra Geom. 3(1), 128-151 (2019)

Ben-Tal, A., Nemirovski, A.: Lectures on Modern Convex Optimization: Analysis, Algorithms and Engineering Applications. SIAM, Philadelphia (2001)

Bochnak, J., Coste, M., Roy, M.-F.: Real Algebraic Geometry. Ergebnisse der Mathematik und ihrer Grenzgebiete, vol. 36. Springer, Berlin (1998)

Chandrasekaran, V., Shah, P.: Relative entropy relaxations for signomial optimization. SIAM J. Optim. 26(2), 1147-1173 (2016)

Dressler, M., Kurpisz, A., de Wolff, T.: Optimization over the Boolean hypercube via sums of nonnegative circuit polynomials. In: Potapov, I., Spirakis, P. G., Worrell, J. (eds.) Proc. Mathematical Foundations of Computer Sciences (MFCS), Liverpool, LIPIcs, Schloss Dagstuhl, vol. 117, pp. 82:1-82:17 (2018a)

Dressler, M., Naumann, H., Theobald, T.: The dual cone of sums of non-negative circuit polynomials. Adv. Geom. (2018b). arXiv: 1809.07648

Forsgård, J., de Wolff, T.: The algebraic boundary of the SONC cone (2019). arXiv:1905.04776

Iliman, S., de Wolff, T.: Amoebas, nonnegative polynomials and sums of squares supported on circuits. Res. Math. Sci. 3(1), 9 (2016)

Karaca, O., Darivianakis, G., Beuchat, P., Georghiou, A., Lygeros, J.: The REPOP toolbox: tackling polynomial optimization using relative entropy relaxations. In: 20th IFAC World Congress, IFAC PapersOnLine, vol. 50(1), pp. 11652-11657. Elsevier (2017)

Katthän, L., Naumann, H., Theobald, T.: A unified framework of SAGE and SONC polynomials and its duality theory (2019). arXiv:1903.08966

Lasserre, J.B.: Moments, Positive Polynomials and their Applications. Imperial College Press Optimization Series, vol. 1. Imperial College Press, London (2010)

Laurent, M.: Sums of squares, moment matrices and optimization over polynomials. In: Putinar, M., Sullivant, S. (eds.) Emerging Applications Of Algebraic Geometry, IMA Vol. Math. Appl., vol. 149, pp. 157-270. Springer, New York (2009)

Marshall, M.: Positive Polynomials and Sums of Squares. Mathematical Surveys and Monographs, vol. 146. American Mathematical Society, Providence (2008)

Murray, R., Chandrasekaran, V., Wierman, A.: Newton polytopes and relative entropy optimization (2018). arXiv: 1810.01614

Murray, R., Chandrasekaran, V., Wierman, A.: Signomial and polynomial optimization via relative entropy and partial dualization (2019). arXiv:1907.00814

Nesterov, Y., Nemirovski, A.: Interior-Point Polynomial Algorithms in Convex Programming. SIAM, Philadelphia (1994)

Papp, D.: Duality of sum of nonnegative circuit polynomials and optimal SONC bounds (2019). arXiv: 1912.04718

Prestel, A., Delzell, C.N.: Positive Polynomials. Springer Monographs in Mathematics. Springer, Berlin (2001)

Reznick, B.: Forms derived from the arithmetic-geometric inequality. Math. Ann. 283(3), 431-464 (1989)

Wang, J.: Nonnegative polynomials and circuit polynomials (2018). arXiv:1804.09455

Wang, J., Magron, V.: Second-order cone representations of SONC cones (2019). arXiv:1906.06179

Publisher's Note Springer Nature remains neutral with regard to jurisdictional claims in published maps and institutional affiliations. 